\title{
CALIDAD DE VIDA Y COMUNICACIÓN FAMILIAR EN MADRES ADOLESCENTES
}

\author{
Dr. Mario Bulnes Bedón* \\ Dr. Carlos Ponce Díaz \\ Ps. Rosa Huerta Rosales \\ Ps. Renato Santivañez Olulo \\ Ps. Marcelino Riveros Quiroz \\ Ps. Jaime Aliaga Tovar \\ Ps. Jorge Hidalgo Zerpa
}

\begin{abstract}
RESUMEN
Se presenta un informe sobre una investigación con una muestra de 123 madres adolescentes de diversas instituciones hospitalarias estatales de Lima para evaluar el grado de relación entre las variables calidad de vida. Comunicación familiar y la condición de madre adolescente.
\end{abstract}

Palabras clave: Calidad de Vida. comunicación familiar. apertura a la comunicación; problema en la comunicación. madre-adolescente .

\begin{abstract}
It presents a report on a research with a sample of 123 adolescent mothers from different hospitalary institutions of Lima. It assesses the relation degrees between life style, familiar communication and the adolescent mother condition variables.
\end{abstract}

Key words: Life style. familiar communication, communication opening. communication problems. adolescent mother.

* Director de la Unidad de Post-Grado de la Facultad de Psicología de la UNMSM. 
El propósito principal de la investigación fue evaluar las relaciones entre comunicación familiar, calidad de vida y la condición de madre adolescente. Se buscó determinar si la maternidad en la adolescencia influiría sobre la calidad de vida y si es el resultado de una deficiente comunicación entre los padres y el adolescente.

Un aspecto de la problemática de la adolescencia, que es entendida como la etapa del ciclo vital que abarca desde los 14 ó 15 hasta los 17 ó 18 años de edad, es la expresión desembozada del impulso sexual. La adolescencia es una fase de rápida maduración biofísica y psicológica.

Un problema que dicha etapa trae consigo lo representa la urgencia de una integración exitosa de la sexualidad con otros aspectos. Por ejemplo, con el surgimiento del sentido del sí mismo, del self o del yo. Y esto debería ocurrir sin conflictos, represión o ansiedad.

El impulso sexual puede parecer, para el adolescente, algo mezclado con la necesidad de amor, con la autoestima o la confianza o con el mismo amor. Para unos, ésta sería una respuesta «natural», propiciada a su vez por una «educación liberal». Pero, para otros, la búsqueda de actividad sexual no sería más que búsqueda de afecto, de reconocimiento o de aceptación, ya sea para huir de la presión de los padres o, como una consecuencia de esto, para rebelarse contra ellos.

En la década de los 90 se incrementó tanto el interés en los investigadores cuanto la desazón social por la cuasi epidémica ocurrencia de embarazos en adolescentes, no sólo en Latinoamérica, sino en todo el mundo. Según la población estimada, el Perú en 1997 era de 24'371.043 habitantes, de los cuales e136\% del total de mujeres en edad reproductiva son precisamente adolescentes.

Según la encuesta Demográfica y de Salud Familiar de 1996, la tasa global de fecundidad en el país ha disminuido de 4.9 hijos por mujer a 3.5 en un lapso de 15 años. Del mismo modo, la tasa específica de fecundidad para todos los grupos etáreos también ha disminuido, excepto para el grupo de 15 a 19 años, que, más bien, está aumentando. Por otra parte, entre las menores de 15 a 17 años, el porcentaje de mujeres analfabetas alcanza al $4 \%$.

Como se ve, la frecuencia de embarazos en adolescentes exhibe una tendencia a crecer, al punto de ser asumido a la fecha como un problema de salud pública. En realidad, lo es por sus implicaciones epidemiológicas, vale decir, por las tasas de incidencia respectivas, por su repercusión en el crecimiento desorganizado de la población, por los efectos adversos en la salud de la madre adolescente Y del niño y, en fin, por retroalimentar la persistencia de la pobreza.

El embarazo en adolescentes es pernicioso, no sólo por sus implicaciones 
«demográficas», sino por los aspectos más contraproducentes que están representados en el mismo hecho de su ocurrencia temprana. Esto repercute en especial en la adolescente con poca o ninguna escolaridad y con escasos o con carencia absoluta de recursos socioeconómicos.

Por ello se consideró de interés teórico explorar cuál es el contexto social y familiar en que se desenvuelven las adolescentes que llegaron a ser madres y en qué condiciones ocurrió su embarazo. Por esto la investigación se concentró en la calidad de vida y en la comunicación familiar en esos grupos etáreos.

Los objetivos específicos del estudio se resumieron en cinco:

1. Si la calidad de vida se relaciona con la comunicación padre-adolescente;

2. Si la calidad de vida se relaciona con la comunicación madre-adolescente;

3. Si la comunicación padre-adolescente está asociada con la comunicación madreadolescente;

4. Si la condición de madre adolescente repercutirá en la calidad de vida; y

5. Si la comunicación padres-adolescente se relaciona con la condición de madre adolescente.

Usualmente, la calidad de vida se mide a través de dos modelos teóricos distintos. Uno es el modelo objetivo, que parte de datos estadísticos sobre indicadores sociales y económicos especificas, como ingreso, gasto en educación, facilidad de recreación, entre otros, seleccionados por su relación conceptual con la calidad de vida.

Sin embargo, se objeta este modelo pues nada revelaría sobre la percepción personal de las situaciones vitales. De allí que se postule un modelo alterno.

Ése es el modelo subjetivo, que utiliza el concepto de bienestar general como una medida de calidad de vida. Este concepto es el más usado en la mayoría de estudios sobre calidad de vida. La prueba empleada en la investigación es, justamente, una que considera las percepciones subjetivas como relevante en la vida de las personas.

Una característica común de los estudios de la calidad de vida es que éstos elevan la satisfacción en los dominios de la experiencia vital de los individuos. como por ejemplo vida familiar, amigos, domicilio, facilidades de vivienda, educación, empleo, religión, etc. La satisfacción dentro de cada uno de estos dominios es un juicio individual y por lo tanto subjetivo. Nos da una referencia de cómo se percibe la satisfacción de necesidades, aspiraciones e intereses personales en la relación con el entorno.

Frecuentemente, los estudios acerca de calidad de vida relacionan satisfacción individual con dominio especifico de experiencia vital. Vale decir, con una evaluación 
global del sentido total de bienestar o satisfacción vital.

En realidad, no hay consenso en cuanto a qué variables y aspectos evaluar. El estudio mas reciente es el expuesto por Otero (1998), quien establece la relación entre calidad de vida y salud-enfermedad. Se considera que la calidad de vida tiene más un sentido personal y subjetivo, por lo cual dichas estimaciones varían de una persona a otra, e incluso de una sociedad a otra.

Por otro lado, es sostenible que los logros humanos se cristalizan merced a condiciones tales como descanso físico y mental reparador, creación y recreación, actividad lúdica y comunicabilidad en una familia integradora y educativa. A este respecto habría indicadores psicológicos y sociales.

Como indicadores psicológicos tenemos la vida personal y las reacciones ante diferentes experiencias. Como casos de indicadores sociales, tenemos las condiciones objetivas de vida, seguridad social, nivel de empleo, etc.

Por esto último la calidad de vida es, antes bien, una noción abstracta y multidimensional u omniabarcadora. Por tanto, es difícil de medir por la complejidad que encierra.

Así y todo, calidad de vida es un concepto que designa a una idea de bienestar fundamentalmente subjetivo. Sólo el sujeto es el juez de sus propias percepciones.

En cuanto a comunicación familiar, hay una variedad de formulaciones y operacionalizaciones que no permiten una comparación. En algunos casos es tratada como un constructo general; en otros, como aprendizaje de habilidades dentro de la terapia, como un estilo o patrones de interacción; o también en términos de componentes específicos tales como empatía, congruencia e interés, o del entusiasmo de parejas para comunicar en el contexto de una relación interpersonal cercana; incluso como un «autodescubrimiento».

También se ha propuesto un modelo teórico de los sistemas marital y familiar. Es el modelo circumplejo. Éste se concentra en la disminución de cohesión y adaptabilidad familiar. Aduce que la comunicación efectiva facilita el movimiento y mantenimiento del sistema en el nivel deseado (balanceado) en las dos principales dimensiones del modelo. La comunicación inefectiva minimiza e incluso puede detener el movimiento hacia niveles balanceados de adaptabilidad.

Por ello se desarrolló escalas para evaluar el aspecto particular de la comunicación. Una de ellas se empleó en la investigación. Se buscó complementar los estudios que han enfocado la comunicación sólo en la relación entre esposos, pero no la comunicación familiar a partir del testimonio de cada miembro (léase: esposos y adolescentes) del hogar. 


\section{MÉTODO}

La investigación fue de tipo descriptivo-exploratoria y correlacional. Se estudio la situación de madre adolescente desde el punto de vista de calidad de vida y la comunicación padres-adolescente.

\section{Sujetos:}

La muestra estuvo constituida por 128 madres adolescentes. Por tratarse de una muestra disponible su carácter fue en consecuencia no probabilistico.

Los sujetos se obtuvieron de las diferentes instituciones hospitalarias:

Hospital Santa Rosa ....... 49

Maternidad de Lima ........ 24

María Auxiliadora .......... 55

Total..................... 128

De las edades comprendidas entre los 14 años hasta 19 años de edad.

\section{INSTRUMENTO}

Se aplicó la prueba de Calidad de Vida de David H. Olson y Howard L. Barnes y la de Comunicación padres-adolescentes de los mismos autores; a esto se acompañó una ficha socio-económica.

El cuestionario de Calidad de Vida contiene 25 items distribuido en 11 áreas o dominios vitales, como por ejemplo:

- Vida Familiar - Educación

- Amigos - Ocio

- Familia Extensa - Religión

- Salud - Hogar

- Medio de Comunicación

- Bienestar Económico

- Vecindario y Comunidad

Los items son de tipo Likert con las siguientes opciones:

1 Insatisfecho

2 Un poco satisfecho

3 Más o menos satisfecho

4 Bastante satisfecho

5 Completamente satisfecho

El cuestionario de comunicación padres-adolescentes consta de dos formatos. Uno de comunicación con la madre y, el otro, de comunicación con el padre. Cada una de las formas a su vez tiene dos subescalas: problemas de comunicación (10 items) y apertura 
de la comunicación (10 items). Los reactivos son de tipo Likert con cinco opciones y los siguientes descriptores:

1 Marcado desacuerdo

2 Moderado desacuerdo

$3 \mathrm{Ni}$ de acuerdo ni en desacuerdo

4 Moderado acuerdo

5 Marcado acuerdo

En ambas pruebas se hizo el análisis respectivo para evaluar la aplicabilidad de las mismas, procediéndose a administrarlas a una muestra piloto de 50 jóvenes con edades de 17 a 23 años. Para ello se fijó los siguientes objetivos:

1. Correlacionar los reactivos del cuestionario Calidad de Vida con su puntaje total (correlación item-test, rit).

2. Correlacionar los reactivos del cuestionario de Comunicación Padres -Adolescentes (formato madre y formato padre con sus respectivos puntajes totales) por medio de la correlación Ítem-test ( rit).

3. Correlacionar entre sí y comparar los puntajes de los formatos comunicación adolescente-madre y adolescente-padre.

Comparar los puntajes medios en las subescalas relativas a los problemas de comunicación y apertura en la comunicación de los dos formatos en conjunto y por separado.

5. Calcular los coeficientes de confiabilidad de ambos cuestionarios.

6. Interrelacionar los cuestionarios.

La evaluación del análisis de los reactivos indica que tres de los elementos del cuestionario de Calidad de Vida no demuestran tener un valor discriminatorio o de validez. Ellos son los siguientes:

Su vida Familiar: Ítem 2

sus hermanos $(\mathrm{x}=3.88)$

Hogar: Item 8.

Sus actuales condiciones de vivienda $(\mathrm{x}=3.88)$

Vecindario: Item 23.

La seguridad en su comunidad $(\mathrm{x}=2.60)$ 


\section{Cuadro $\mathbf{N}^{\circ} 1$}

Análisis de Items (Correlación ItemTest) de la Escala de Calidad de vida.

\begin{tabular}{|c|l|l|l|}
\hline $\mathbf{I T E M}$ & $\mathbf{X}$ & $\mathbf{S}$ & $\mathbf{R I T}$ \\
\hline 1 & 3.84 & 0.92 & 0.56 \\
2 & 3.88 & 0.65 & 0.00 \\
3 & 4.24 & 1.34 & 020 \\
4 & 4.16 & 0.73 & 0.38 \\
5 & 3.20 & 1.13 & 0.66 \\
6 & 3.88 & 1.18 & 0.40 \\
7 & 3.20 & 1.13 & 0.64 \\
$\mathbf{8}$ & $\mathbf{3 . 8 8}$ & $\mathbf{0 . 9 1}$ & $\mathbf{0 . 1 2}$ \\
9 & 3.96 & 0.77 & 0.32 \\
10 & 4.04 & 0.92 & 0.50 \\
11 & 3.24 & 1.11 & 026 \\
12 & 3.48 & 0.98 & 0.60 \\
13 & 3.40 & 0.98 & 0.57 \\
14 & 3.32 & 0.88 & 0.37 \\
15 & 3.44 & 1.06 & 0.59 \\
16 & 3.12 & 1.21 & 0.25 \\
17 & 3.60 & 1.02 & 0.66 \\
18 & 3.16 & 0.88 & 0.31 \\
19 & 3.64 & 0.88 & 0.66 \\
20 & 3.56 & 0.98 & 0.42 \\
21 & 324 & 0.91 & 0.35 \\
22 & 3.32 & 1.08 & 0.42 \\
23 & 2.60 & 0.89 & 0.01 \\
24 & 3.08 & 1.23 & 0.23 \\
25 & 3.08 & 1.29 & 0.27 \\
& & & \\
\hline
\end{tabular}

\section{Cuadro $\mathbf{N}^{\circ} 2$}

Análisis de Items (Correlación itemtest) de la Escala de Comunicación (Adolescente-Madre)

\begin{tabular}{|c|c|c|c|}
\hline ITEM & $\mathrm{X}$ & $\mathrm{S}$ & RIT \\
\hline 1 & 4.16 & 1.05 & 0.42 \\
2 & 3.00 & 1.52 & 0.46 \\
3 & 3.92 & 1.09 & 0.63 \\
4 & 324 & 1.39 & 0.67 \\
5 & 3.36 & 1.41 & 0.50 \\
6 & 3.68 & 1.12 & 0.59 \\
7 & 3.76 & 1.03 & 0.77 \\
8 & 3.96 & 0.99 & 0.57 \\
9 & 4.08 & 0.84 & 029 \\
10 & 2.84 & 125 & 0.51 \\
$\mathbf{1 1}(+)$ & $\mathbf{2 . 3 4}$ & $\mathbf{1 . 2 8}$ & $\mathbf{- 0 . 1 9}$ \\
12 & 3.36 & 126 & 0.30 \\
13 & 4.12 & 1.01 & 0.75 \\
14 & 3.80 & 1.20 & 0.58 \\
15 & 3.12 & 1.45 & 0.71 \\
16 & 3.48 & 1.24 & 0.33 \\
17 & 3.20 & 1.30 & 0.45 \\
18 & 3.64 & 1.23 & 0.74 \\
19 & 3.04 & 1.46 & 0.39 \\
20 & 3.24 & 1.48 & 0.76 \\
& & & \\
\hline
\end{tabular}

(+) Coeficiente rit no significativo. 
La evaluación del análisis de los items indica que en esta muestra el reactivo señalado con el número 11 «Soy muy cuidadosa con lo que le digo a mi madre» ( $\mathrm{x}=2.32$; $\mathrm{DS}=$ 1.28 , calificación invertida) no discrimina a las respondientes de buena comunicación con su madre. La tendencia es a responder en dirección a la opción (4), Moderado Acuerdo, que indicaría reserva en ciertos temas, a contrapelo de las tendencias de respuesta a los otros ítemes. Debe señalarse que este elemento pertenece a la subescala de Problemas de comunicación.

La observación del cuadro también indica que hay tres reactivos que alcanzan un promedio de cuatro puntos (Moderado Acuerdo):

(1) Puedo discutir mis creencias con mi madre sin sentirme cohibido;

(9) Yo le demuestro afecto a mi madre abiertamente

(13) Cuando le hago preguntas, recibo respuestas francas de mi madre.

Estos tres reactivos pertenecen a la subescala de Apertura de la comunicación. La contraposición de estos reactivos con el $\mathrm{N}^{\circ} 11$ plantea interesantes interrogantes acerca de la amplitud de la comunicación de hija a madre.

\section{Cuadro $\mathbf{N}^{\circ} 3$ \\ Análisis de Ítems (Correlación Item Test) de la Escala de Comunicación (Adolescente Padres)}

\begin{tabular}{|c|c|c|c|}
\hline ITEM & $\mathbf{X}$ & $\mathbf{S}$ & RIT \\
\hline 1 & 3.64 & 1.49 & 0.23 \\
2 & 3.64 & 1.47 & 0.25 \\
3 & 3.20 & 1.20 & 0.61 \\
$\mathbf{4}$ & $\mathbf{3 . 2 8}$ & $\mathbf{1 . 4 6}$ & $\mathbf{0 . 1 8}$ \\
5 & 3.20 & 1.55 & 0.47 \\
6 & 2.60 & 1.30 & 0.30 \\
7 & 3.52 & 1.24 & 0.48 \\
8 & 3.12 & 1.61 & 0.39 \\
9 & 3.48 & 1.39 & 0.74 \\
10 & 2.48 & 1.44 & 0.31 \\
11 & $\mathbf{2 . 8 8}$ & $\mathbf{1 . 3 4}$ & $\mathbf{0 . 3 3}$ \\
12 & 3.12 & 1.27 & 0.44 \\
$\mathbf{1 3}$ & $\mathbf{4 . 2 0}$ & $\mathbf{1 . 1 1}$ & $\mathbf{- 0 . 0 6}$ \\
14 & 3.52 & 1.45 & 0.35 \\
15 & 2.56 & 1.42 & 0.57 \\
16 & 3.20 & 1.44 & 0.55 \\
17 & 2.80 & 1.47 & 0.53 \\
18 & 3.04 & 1.51 & 0.59 \\
19 & 3.60 & 1.39 & 0.46 \\
20 & 2.92 & 1.35 & 0.17 \\
\hline
\end{tabular}

(+) Coeficiente rit no significativo.

El análisis indica que dos items no alcanzan correlaciones significativas (rit de por lo menos 0.20), el número $4 \mathrm{y}$ el número 13. Lo concerniente a este último ítem es lo que más impresiona. En efecto, a pesar de tener el promedio más alto de todo el formato (4) (Moderado Acuerdo), también logra una correlación de 0 con el puntaje total de la escala 
(su valor discriminativo es nulo). Estos elementos son los siguientes:

(4) A veces temo pedirle a mi padre lo que deseo;

(13) Cuando le hago preguntas recibo respuestas francas de mi padre

El reactivo 4 pertenece a la subescala de problemas de la comunicación en tanto que el 13 a la de Apertura de la comunicación:

\section{DATOS PSICOSOCIALES}

En relación a la muestra misma madre-adolescente y tomando en cuenta los resultados del cuestionario de datos psicosociales podemos observar lo siguiente:

El $49 \%$ tiene estudios imcompletos (Ver Tabla $\boldsymbol{N}^{\boldsymbol{0}} \mathbf{4}$ )

Tabla $\mathbf{N}^{\circ} 4$

Grado de Instrucción

\begin{tabular}{|l|c|c|}
\hline Grado de Instrucción & f & \% \\
\hline Primaria Completa & 17 & 13 \\
Primaria Incompleta, & 10 & 8 \\
Secundaria Completa & 52 & 41 \\
Secundaria Incompleta. & 46 & 36 \\
Universidad Incompleta & 3 & 2 \\
\hline TOTAL & $\mathbf{1 2 8}$ & $\mathbf{1 0 0}$ \\
\hline
\end{tabular}

La mayor frecuencia de edades de las adolescentes recae entre los 16 y 19 años, siendo la media 17 años (ver Tabla $N^{o}$ 5)

Tabla $\mathbf{N}^{\circ} 5$

Edad del Adolescente

\begin{tabular}{|c|c|c|}
\hline Años & f & \% \\
\hline 14 & 3 & 2 \\
15 & 10 & 8 \\
16 & 20 & 16 \\
17 & 40 & 31 \\
18 & 31 & 24 \\
19 & 24 & 19 \\
\hline TOTAL & $\mathbf{1 2 8}$ & $\mathbf{1 0 0}$ \\
\hline
\end{tabular}

La mayor frecuencia de la edad de los padres del hijo de la adolescente se da entre los 16 y 20 años de edad, pero si bien la frecuencia es menor entre las edades de 26 a 40 años, cualitativamente es importante (Ver Tabla $\boldsymbol{N}^{\circ}$ 6) 
Tabla $\mathbf{N}^{\circ} 6$

Edad del padre del hijo de la adolescente madre

\begin{tabular}{|c|r|c|}
\hline EDAD & f & \% \\
\hline $16-20$ & 67 & 52 \\
$21-25$ & 48 & 38 \\
$26-30$ & 8 & 6 \\
$31-40$ & 5 & 4 \\
\hline TOTAL & $\mathbf{1 2 8}$ & $\mathbf{1 0 0}$ \\
\hline
\end{tabular}

El 93\% no mantiene una relación de parentesco con el padre de su hijo; sin embargo, el $7 \%$ mantiene una relación de parentesco como primo, hermano, tío y padre (Ver Tabla $\left.N^{\circ} 7\right)$

Tabla $\mathbf{N}^{0} 7$

Parentesco con el padre de su hijo

\begin{tabular}{|l|c|c|}
\hline PARENTESCO & f & \% \\
\hline Primo & 4 & 3 \\
Hermano & 1 & 1 \\
Tío & 2 & 2 \\
Padre & 1 & 1 \\
No parentesco & 120 & 93 \\
\hline TOTAL & $\mathbf{1 2 8}$ & $\mathbf{1 0 0}$ \\
\hline
\end{tabular}

El $51 \%$ presenta una relación conyugal correspondiente a sus padres de manera informal y critica (conviviente, divorciados, separados). Si bien e143\% presenta una relación formal ( casados), habría que analizar la dinámica familiar (Ver Tabla $\boldsymbol{N}^{\circ} 8$ ).

Tabla $\mathbf{N}^{\circ} 8$

Estado Civil de los padres de la Adolescente

\begin{tabular}{|l|r|r|}
\hline ESTADO CIVIL & F & \multicolumn{1}{|c|}{} \\
\hline Conviviente & 10 & 31 \\
Casados & 55 & 43 \\
Divorciados & 4 & 3 \\
Separados & 22 & 17 \\
Viudo(a) & 7 & 5 \\
\hline TOTAL & $\mathbf{1 2 8}$ & $\mathbf{9 9}$ \\
\hline
\end{tabular}

El $62 \%$ de los adolescentes viene de familia numerosa, pudiendo ésta ser de más de tres hermanos hasta incluso diez (Ver Tabla $\left.\boldsymbol{N}^{\circ} \mathbf{9}\right)$.

El 83\% de madres adolescentes no continúa: estudiando (Ver Tabla $\mathbf{N}^{\circ} \mathbf{1 0}$ )

El 79\% de adolescente no trabaja y el $21 \%$, sí. (Ver Tabla $\left.\boldsymbol{N}^{\circ} \mathbf{1 1}\right)$ 


\section{Tabla $\mathbf{N}^{0} 9$}

Número de hermanos que viven con la adolescente

\begin{tabular}{|c|r|r|}
\hline N $^{\circ}$ HERM. & $\mathbf{f}$ & $\mathbf{\%}$ \\
\hline 1 & 5 & 4 \\
2 & 19 & 15 \\
3 & 24 & 19 \\
4 & 23 & 18 \\
5 & 19 & 15 \\
6 & 18 & 14 \\
7 & 10 & 8 \\
8 & 5 & 4 \\
9 & 2 & 1 \\
10 & 3 & 2 \\
\hline TOTAL & $\mathbf{1 2 8}$ & $\mathbf{1 0 0}$ \\
\hline
\end{tabular}

Tabla $\mathbf{N}^{\circ} \mathbf{1 0}$

Situación escolar de la Madre-Adolescente

\begin{tabular}{|c|c|c|}
\hline ESTUDIA & f & \% \\
\hline NO & 106 & 83 \\
SI & 22 & 17 \\
\hline TOTAL & $\mathbf{1 2 8}$ & $\mathbf{1 0 0}$ \\
\hline
\end{tabular}

Tabla $\mathbf{N}^{0} \mathbf{1 1}$

Situación Laboral de la Madre-Adolescente

\begin{tabular}{|c|c|c|}
\hline TRABAJA & f & \% \\
\hline NO & 101 & 79 \\
SI & 27 & 21 \\
\hline TOTAL & $\mathbf{1 2 8}$ & $\mathbf{1 0 0}$ \\
\hline
\end{tabular}

\section{RESULTADOS}

\section{Cuadro No 12}

Media y Desviación Estandar de las pruebas de Comunicación Padres-Adolescente y Calidad de Vida

\begin{tabular}{|l|c|c|l|}
\hline Variable & Casos & Media & Desv.Est \\
\hline TOTCPAP* & 128 & 57.7266 & 21.7218 \\
TOTCPAM & 128 & 63.1016 & 19.6544 \\
TOTCV* & 128 & 77.6484 & 20.9095 \\
\hline
\end{tabular}

* TOCPAP: Total puntaje de comunicación padre-adolescente versión padre *TOCPAM: Total puntaje de comunicación padre-adolescente versión madre

*TOTALCV: Total puntaje de la prueba sobre Calidad de Vida 


\section{Cuadro $\mathbf{N}^{\circ} 13$ \\ Índices de Correlación entre calidad de vida de la Madre-Adolescente y la calidad de comunicación con cada uno de sus padres}

\begin{tabular}{|c|c|c|c|}
\hline & $\begin{array}{l}\text { Calidad de } \\
\text { Vida }\end{array}$ & $\begin{array}{c}\text { Comunico } \\
\text { Padre-Adol } \\
\text { (Padre) }\end{array}$ & $\begin{array}{l}\text { Comunico } \\
\text { Padre-Adol } \\
\text { (Madre) }\end{array}$ \\
\hline  & & $0.29 *$ & $0.28 *$ \\
\hline 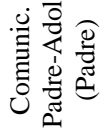 & $0.29 *$ & & $0.50 *$ \\
\hline 总总 & $0.28^{*}$ & $0.50 *$ & \\
\hline
\end{tabular}

$N=128$ (índice de correlación de Pearson al 0.05 de confiabilidad)

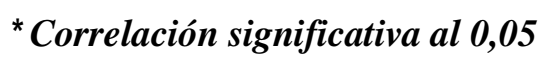

* Existe una influencia débil (aún cuando significativa) entre el índice de satisfacción con la calidad de vida de la madre adolescente y la cantidad de apertura y experiencia comunicativa con cada uno de sus padres.

* Existe una influencia moderada mutua entre la cantidad de apertura y experiencia comunicativa de la madre adolescente con cada uno de sus padres.

\section{Cuadro $\mathrm{N}^{\circ} 14$}

Diferencia entre puntajes promedios obtenidos en la escala de comunicación PadreAdolescente (Madre) y escala de comunicación Padre-Adolescente (Padre)

\begin{tabular}{|c|c|c|}
\hline & \multicolumn{2}{|c|}{ Muestras } \\
\hline & $\begin{array}{c}\text { Comunico } \\
\text { Padre-Adol } \\
\text { (Padre) } \\
\mathrm{N}=128\end{array}$ & $\begin{array}{c}\text { Comunico } \\
\text { Padre-Adol } \\
\text { (Madre) } \\
\mathrm{N}=128\end{array}$ \\
\hline $\begin{array}{c}\text { Media } \\
\text { Aritmeticas }\end{array}$ & $61.33(\mathrm{pc} 28)$ & $54.42(\mathrm{Pc} 19)$ \\
\hline t Teórica & \multicolumn{2}{|c|}{ \pm 2.00} \\
\hline t Calculada & \multicolumn{2}{|c|}{-3.22372} \\
\hline $\begin{array}{l}\text { Conclusión } \\
(\&=0.05)\end{array}$ & \multicolumn{2}{|c|}{$\begin{array}{l}\text { Si hay diferencias } \\
\text { significativas }\end{array}$} \\
\hline
\end{tabular}

La cantidad de apertura y experiencia comunicativa de las madres adolescentes con ambas figuras paternas, es ostensiblemente muy limitada. Pero, comparativamente existe un nivel significativamente mayor a favor de la figura materna. 


\section{Cuadro $\mathbf{N}^{\circ} 15$ \\ Diferencia entre puntajes promedios obtenidos en la escala de comunicación Padre- Adolescente (Padre) por Madres-Adolescentes según el Quartil que se ubican en la Escala de calidad de Vida}

\begin{tabular}{|c|c|c|}
\hline & \multicolumn{2}{|r|}{ Muestras } \\
\hline & $\begin{array}{c}\text { Comunico } \\
\text { Padre-Adol (Padre) } \\
\text { Q1Cal. de Vid. } \\
\mathrm{N}=32\end{array}$ & $\begin{array}{c}\text { Comunico } \\
\text { Padre-Adol (Padre). } \\
\text { Q3 Cal. de Vid } \\
\text { N=33 }\end{array}$ \\
\hline Media Aritmetica & 47.12(Pc 10) & $61.24(\operatorname{Pc} 38)$ \\
\hline t Teórica & \multicolumn{2}{|c|}{ \pm 2.00} \\
\hline t Calculada & \multicolumn{2}{|c|}{-3.46403} \\
\hline $\begin{array}{l}\text { Conclusión } \\
(\boldsymbol{\&}=0.05)\end{array}$ & \multicolumn{2}{|c|}{$\begin{array}{l}\text { Si hay diferencias } \\
\text { significativas }\end{array}$} \\
\hline
\end{tabular}

La cantidad de apertura y experiencia comunicativa de las madres adolescentes con la figura paterna, es ostensiblemente muy limitada. Pero, comparativamente existe un nivel significativamente mayor entre las madres adolescentes que tienen un mejor índice de satisfacción con su calidad de vida.

\section{Cuadro $\mathrm{N}^{\circ} 16$}

Diferencia entre puntajes promedios obtenidos en la escala de comunicación PadreAdolescente (Madre) por Madres-Adolescentes según el Quartil que se ubican en la escala de calidad de vida

\begin{tabular}{|c|c|c|}
\hline & \multicolumn{2}{|r|}{ Muestras } \\
\hline & $\begin{array}{c}\text { Comunico } \\
\text { Padre-Adol. (Madre) } \\
\text { Q1Cal. de Vid. } \\
\text { N=32 }\end{array}$ & $\begin{array}{c}\text { Comunico } \\
\text { Padre-Adol (Madre). } \\
\text { Q3 Cal. de Vid } \\
\text { N=33 }\end{array}$ \\
\hline Media Aritmética & $53.43(\mathrm{pc} 12)$ & $65.48(\mathrm{pc} 43)$ \\
\hline t Teórica & \multicolumn{2}{|c|}{ \pm 2.00} \\
\hline t Calculada & \multicolumn{2}{|c|}{-3.28163} \\
\hline Conclusión $(\&=0.05)$ & \multicolumn{2}{|c|}{ Si hay diferencias significativas } \\
\hline
\end{tabular}

La cantidad de apertura y experiencia comunicativa de las madres adolescentes con la figura materna, es ostensiblemente muy limitada. Pero, comparativamente existe un nivel significativamente mayor entre las madres adolescentes que tienen un mejor índice de satisfacción con su calidad de vida. 


\section{Cuadro $\mathbf{N}^{\circ} 17$}

Diferencia entre puntajes promedios obtenidos en la escala de calidad de vida por MadresAdolescentes según el Quartil que se ubican en la escala de comunicación Padre-Adolescente (Padre)

\begin{tabular}{|c|c|c|}
\hline & \multicolumn{2}{|c|}{ Muestras } \\
\hline & $\begin{array}{c}\text { Comunico } \\
\text { Padre-Adol. (Padre) } \\
\text { Q1 Cal. de Vid. } \\
\text { N=32 }\end{array}$ & $\begin{array}{c}\text { Comunico } \\
\text { Padre-Adol. (Padre). } \\
\text { Q3 Cal. de Vid } \\
\text { N=32 }\end{array}$ \\
\hline Media Aritmetic & $66.96(\mathrm{pc} 11)$ & $83.5(\mathrm{pc} 35)$ \\
\hline t Teórica & \multicolumn{2}{|c|}{ \pm 2.00} \\
\hline t CalcuIada & \multicolumn{2}{|c|}{-3.39654} \\
\hline Conclusión $(\&=0.05)$ & \multicolumn{2}{|c|}{ Si hay diferencias significativas } \\
\hline
\end{tabular}

El índice de satisfacción con su calidad de vida de las madres adolescentes, es ostensiblemente muy bajo. Pero, comparativamente, existe un índice significativamente mayor entre las madresadolescentes que tienen una mayor cantidad de apertura y experiencia comunicativa con la figura paterna.

\section{Cuadro $\mathrm{N}^{\circ} 18$}

Diferencia entre puntajes promedios obtenidos en la escala de calidad de vida por MadresAdolescentes según el Quartil que se ubican en la escala de comunicación Padre-Adolescente (Madre)

\begin{tabular}{|c|c|c|}
\hline & \multicolumn{2}{|c|}{ Muestras } \\
\hline & $\begin{array}{c}\text { Comunico } \\
\text { Padre-Adol. (Madre) } \\
\text { Q 1 Cal. de Vid. } \\
\mathrm{N}=32 \\
\end{array}$ & $\begin{array}{c}\text { Comunico } \\
\text { Padre-Adol.(Madre). } \\
\text { Q3 Cal. de Vid } \\
\text { N=34 } \\
\end{array}$ \\
\hline Media Aritmética & 71.34(Pc I1) & $82.79(\mathrm{Pc} 35)$ \\
\hline T Teórica & \multicolumn{2}{|c|}{ \pm 2.00} \\
\hline t Calculada & \multicolumn{2}{|c|}{-2.20035} \\
\hline Conclusión $(\&=0.05)$ & \multicolumn{2}{|c|}{ Si hay diferencias significativas } \\
\hline
\end{tabular}

El índice de satisfacción con su calidad de vida de las madres adolescentes, es ostensiblemente muy bajo. Pero, comparativamente existe un índice significativamente mayor entre las madres adolescentes que tienen una mayor cantidad de apertura y experiencia comunicativa con la figura materna.

\section{INTERPRETACION}

Todo hace pensar que el factor Calidad de Vida y el factor Comunicación Padresadolescentes mantienen una relativa dependencia, en la medida que su correlación es baja.

Se observa una correlación moderada, aunque significativa entre el factor Comunicación Madre-Adolescente y el factor Comunicación Padre-Adolescente. Pero, 
si nos detenemos en la calidad de la comunicación ésta, es más favorable con la madre de la adolescente y muy limitada con el padre. Esto nos haría pensar en la importancia de la madre como elemento afectivo y la limitación del padre para incorporarse a esa disposición.

Se puede observar que cualitativamente se ve afectada la calidad de vida por el tipo de comunicación que establecen los padres.

El índice en la calidad de vida es evidentemente muy bajo en la madreadolescente, y probablemente acompañado de una deficiente autoestima.

\section{CONCLUSIONES}

1. Se encontró relación entre los factores Calidad de Vida y Comunicación padreadolescente.

2. Se encontró una relación entre los factores Calidad de Vida y Comunicación madreadolescente.

3. Se encontró relación entre los factores Comunicación padre-adolescente y Comunicación madre-adolescente

4. Se encontró que el factor Calidad de Vida de las madres-adolescentes presenta un índice bajo.

5. El nivel de comunicación del padre con la madre-adolescente es bajo

\section{BIBLIOGRAFIA}

Congreso de la República (1998) Jóvenes en Cifras. Perú 1998. Lima Perú.

Convenio de Cooperación Técnica para la Atención Primaria de Salud con la Comunidad Educativa. Ministerio de Salud Ministerio de Educación. Perú 1992-1997.

Donas Solum, (1996) Marco Epidemiológico conceptual de la salud integral del adolescente. OPS, San José de Costa Rica.

Ferrando D., (1991) Los Jóvenes en el Perú: Opiniones, actitudes y valores. Encuesta Nacional de Hogares, CEDRO, Lima Perú. INEI (1998) Estado de la Población Peruana: 1998. Situación de la Población Joven y de la Tercera Edad. Lima Perú.

López L., (1996). Salud Reproductiva de los adolescentes. Ponencia 1 Congreso Internacional Salud reproductiva y Sociedad. UPCH, PROSAR, Lima Perú.

Ministerio de Salud (1998) Plan Nacional para la atención integral de la Salud del Escolar y Adolescente 1997-2001. Lima Perú.

Otero, Haydée (1998) Calidad de Vida y Enfermedad. En: Revista de la Facultad de Psicología de la Universidad Nacional Mayor de San Marcos. Pg. 125 - 133.

UNFPA (1997) Fondo de Población de las Naciones Unidas: Descripción, Funcionamiento y Labor realizada en el Perú. 